\title{
THE CODIMENSION OF THE BOUNDARY OF A LATTICE IDEAL ${ }^{1}$
}

\author{
J. W. LEA, JR.
}

ABSTRACT. In a compact connected topological lattice of finite codimension $n$, the boundary of a proper principal ideal has codimension less than $n$. It follows that the boundaries of proper intervals also have codimension less than $n$.

In a topological lattice $L$ the boundary of a principal (dual) ideal is a join (meet) subsemilattice of $L$ [1, Lemma 5]. The purpose of this paper is to show that this semilattice has codimension less than $n$ whenever $L$ is a compact, connected topological lattice of codimension $n$. Note that such a lattice has breadth $n$ [5, Corollary 2.4].

If $S$ is a semilattice and $x \in S$, then $M(x)=\{y \in S: x \leqq y\}$; the set $L(x)$ is defined dually; if $x \leqq y$, then $[x, y]=M(x) \cap L(y)$. The interior and the closure of $A$ are denoted by $A^{\circ}$ and $A^{*}$ respectively. The boundary of $A$ is $F(A)=A^{*} \backslash A^{\circ}$. If $a \in S$ a topological semilattice and $x \in F(M(a))$, then $[a, x] \subset F(M(a))$.

Lemma. Let $L$ be a compact, connected topological lattice of finite codimension. If $a \in L$, then $F(M(a))$ is locally connected.

Proof. Let $x \in F(M(a))$ and let $U$ be an open subset of $F(M(a))$ which contains $x$. It is known that $L$ has a basis of open convex sublattices [6, Theorem 5]. Hence we may choose an open convex sublattice $V$ containing $x$ such that $V \cap F(M(a)) \subset U$. Let $p, q \in V \cap F(M(a))$; then the connected set $[p \wedge q, p] \cup[p \wedge q, q]$ is contained in $V \cap F(M(a))$. Thus $V \cap F(M(a))$ is a connected open subset of $F(M(a))$ which contains $x$ and which is contained in $U$. Therefore $F(M(a))$ is locally connected.

We shall state and prove the main result in terms of principal dual ideals.

THEOREM. Let $L$ be a compact, connected topological lattice of finite codimension $n$ and let $a \in L \backslash\{0,1\}$. Then the codimension of $F(M(a))$ is less than $n$.

Received by the editors May 8, 1973.

AMS (MOS) subject classifications (1970). Primary 06A20, 54F05.

Key words and phrases. Compact semilattice, compact lattice, ideal.

${ }^{1}$ A portion of this paper appears in the author's dissertation presented to the Graduate School of Louisiana State University and directed by Professor J. D. Lawson. 
Proof. Since the result clearly holds for $n \leqq 1$, we shall assume $n>1$.

We shall first show that $x \in F(M(a))$ implies $[a, x]$ has codimension less than $n$. Let $x \in F(M(a))$ and let $U(a)=L \backslash[L(a) \cup M(a)]$. If $U(a)=\varnothing$, then $a$ is a cut point of $L$ [2, Theorem 1]. Thus $a=x$ and $[a, x]=\{a\}$ has codimension 0 .

Suppose $U(a) \neq \varnothing$ and $x \neq a$. We shall construct an increasing net in $L(x) \cap U(a)$ which converges to $x$. Since $x \notin L(a)$ we may choose an open set $V$ containing $x$ such that $V \cap L(a)=\varnothing$. Now $x \in F(M(a))$ implies $V \cap L \backslash M(a) \neq \varnothing$. Note that $L \backslash M(a)=[L(a) \cup U(a)] \backslash\{a\}$ so that $V \cap U(a) \neq$ $\varnothing$. Thus we may choose a net $\left\{x_{\alpha}^{\prime}\right\}_{\alpha \in \Delta} \subset U(a)$ which converges to $x$. Let $y_{\alpha}=x \wedge x_{\alpha}^{\prime}$ for all $\alpha \in \Delta$. Then $\left\{y_{\alpha}\right\}_{\alpha \in \Delta} \subset L(x)$ and converges to $x$. If $\left\{y_{\alpha}\right\}_{\alpha \in \Gamma} \subset L(a)$ for any cofinal subset $\Gamma \subset \Delta$, then $\left\{\left(y_{\alpha}, a\right)\right\}_{\alpha \in \Gamma}$ converges to $(x, a) \in L \times L$. Since the graph of $\leqq$ is a closed subset of $L \times L$, then $x \leqq a$. But $x \in M(a)$ so that $x=a$ contrary to the choice of $x \neq a$. Hence we may assume $y_{\alpha} \notin L(a)$ for all $\alpha \in \Delta$. If $y_{\alpha} \in M(a)$, then $a \leqq y_{\alpha}=x \wedge x_{\alpha}^{\prime} \leqq x_{\alpha}^{\prime}$ contrary to $a$ 主敉. Thus $\left\{y_{\alpha}\right\}_{\alpha \in \Delta} \subset L(x) \cap U(a)$.

Let $x_{\alpha}=\bigwedge_{\beta \geq \alpha} y_{\beta}$ for each $\alpha \in \Delta$. Clearly $\left\{x_{\alpha}\right\}_{\alpha \in \Delta}$ is an increasing net contained in $L(x)$. Let $U$ be an open set containing $x$. There exists a closed sublattice $V \subset U$ such that $x \in V^{\circ}$ [6, Theorem 5]. Since $\left\{y_{\alpha}\right\}_{\alpha \in \Delta}$ converges to $x$, there exists $\beta \in \Delta$ such that $\alpha \geqq \beta$ implies $y_{\alpha} \in V^{\circ}$. Hence $x_{\gamma}=\bigwedge_{\alpha \geqq \gamma} y_{\alpha} \in V$ for all $\gamma \geqq \beta$. Thus $\left\{x_{\alpha}\right\}_{\alpha \in \Delta}$ converges to $x$. That $\left\{x_{\alpha}\right\}_{\alpha \in \Delta} \subset$ $U(a)$ follows just as did the fact that $\left\{y_{\alpha}\right\}_{\alpha \in \Delta} \subset U(a)$. Therefore $\left\{x_{\alpha}\right\}_{\alpha \in \Delta}$ is the required net.

For each $\alpha \in \Delta$, the interval [ $a, a \vee x_{\alpha}$ ] has breadth less than $n$ [7, Lemma 1.1]. Since $\left\{x_{\alpha}\right\}_{\alpha \in \Delta}$ is increasing, $\left\{\left[a, a \vee x_{\alpha}\right]\right\}_{\alpha \in \Delta}$ is a chain. Therefore $\bigcup_{\alpha \in \Delta}\left[a, a \vee x_{\alpha}\right]$ has breadth less than $n$; consequently the breadth of $\left(\bigcup_{\alpha \in \Delta}\left[a, a \vee x_{\alpha}\right]\right) *$ is less than $n$. Since the interval $\left[a, a \vee x_{\alpha}\right]=\left(a \vee x_{\alpha}\right) \wedge M(a)$, it follows that $[a, x]=\left(\bigcup_{\alpha \in \Delta}\left[a, a \vee x_{\alpha}\right]\right)^{*}[3$, Theorem 3]. Thus $[a, x]$ has breadth less than $n$, and since $[a, x]$ is a compact, connected topological lattice, its breadth and its codimension are equal.

The semilattice $F(M(a))$ is a Lawson semilattice, i.e. has a neighborhood basis of subsemilattices [5, Theorem 1.1]. A. Y. W. Lau has shown that any compact, connected, locally connected Lawson semilattice $S$ contains a point $x$ for which the codimension of $S$ and the codimension of $L(x)$ are equal [4, Lemma 5.2]. Thus for some $x \in F(M(a))$ the codimension of $[a, x]$ and the codimension of $F(M(a))$ are equal.

Corollary. Let $L$ be a compact connected topological lattice of finite codimension $n$. If $a, b \in L$ and $a<b$, then the codimension of $F([a, b])$ is less than $n$. Thus $L$ has a basis of open sets whose boundaries have codimension less than $n$. 
Proof. If $a=0$ or $b=1$, then $[a, b]=L(b)$ or $[a, b]=M(a)$. Thus we may assume $a \neq 0$ and $b \neq 1$. It is easy to see that $F([a, b])$ is a closed subset of $F(M(a)) \cup F(L(b))$. By the theorem each of these sets has codimension less than $n$. Thus the codimension of $F([a, b])$ is less than $n$.

Since $L$ has a basis of neighborhoods at each point consisting of intervals $[a, b][6$, Theorem 5], the collection of interiors of these intervals is the desired basis.

\section{REFERENCES}

1. L. W. Anderson, One dimensional topological lattices, Proc. Amer. Math. Soc. 10 (1959), 715-720. MR 21 \#6401.

2. - On the distributivity and simple connectivity of plane topological lattices, Trans. Amer. Math. Soc. 91 (1959), 102-112. MR 21 \#1365.

3. R. J. Koch, On monothetic semigroups, Proc. Amer. Math. Soc. 8 (1957), 397-401. MR 19, 290.

4. A. Y. W. Lau, Small semilattices and costability, Dissertation, University of Houston, Houston, Tex., 1971.

5. J. D. Lawson, The relation of breadth and codimension in topological semilattices. II, Duke Math. J. 38 (1971), 555-559. MR 44 \#125.

6. _ Intrinsic topologies in lattices and semilattices, Pacific J. Math. 44 (1973), 593-602.

7. E. D. Shirley and A. R. Stralka, Homomorphisms on connected topological lattices, Duke Math. J. 38 (1971), 483-490.

Department of Mathematics, Middr.e Tennessee State University, MurfreesBORO, TENNESSEE 37130 